\title{
Stable Rhodopsin/Arrestin Complex Leads to Retinal Degeneration in a Transgenic Mouse Model of Autosomal Dominant Retinitis Pigmentosa
}

\author{
Jiayan Chen, ${ }^{1,2}$ Guang Shi, ${ }^{1}$ Francis A. Concepcion, ${ }^{1,3}$ Guifu Xie, ${ }^{4}$ Daniel Oprian, ${ }^{4}$ and Jeannie Chen ${ }^{1,2,3}$ \\ ${ }^{1}$ Zilkha Neurogenetic Institute, ${ }^{2}$ Neuroscience Graduate Program, and ${ }^{3}$ Department of Cell and Neurobiology and Department of Ophthalmology, Keck \\ School of Medicine, University of Southern California, Los Angeles, California 90033, and ${ }^{4}$ Department of Biochemistry and Volen Center for Complex \\ Systems, Brandeis University, Waltham, Massachusetts 02545
}

\begin{abstract}
Over 100 rhodopsin mutation alleles have been associated with autosomal dominant retinitis pigmentosa (ADRP). These mutations appear to cause photoreceptor cell death through diverse molecular mechanisms. We show that K296E, a rhodopsin mutation associated with ADRP, forms a stable complex with arrestin that is toxic to mouse rod photoreceptors. This cell death pathway appears to be conserved from flies to mammals. A genetics approach to eliminate arrestin unmasked the constitutive activity of K296E and caused photoreceptor cell death through a transducin-dependent mechanism that is similar to light damage. Expressing K296E in the arrestin/ transducin double knock-out background prevented transducin signaling and led to substantially improved retinal morphology but did not fully prevent cell death caused by K296E. The adverse effect of K296E in the arrestin/transducin knock-out background can be mimicked by constant exposure to low light. Furthermore, we found that arrestin binding causes K296E to mislocalize to the wrong cellular compartment. Accumulation of stable rhodopsin/arrestin complex in the inner segment may be an important mechanism for triggering the cell death pathway in the mammalian photoreceptor cell.
\end{abstract}

Key words: photoreceptor; retina; GPCR; retinal degeneration; light damage; blindness

\section{Introduction}

Rhodopsin, a prototypical G-protein-coupled receptor (GPCR), is a light-sensitive visual pigment in retinal rod photoreceptors. Photon absorption causes isomerization of 11-cis-retinal, the chromophore that is covalently attached to the opsin apoprotein. This, in turn, causes conformational changes in the protein moiety and subsequent activation of transducin, the visual G-protein. Like other GPCRs, rhodopsin is deactivated sequentially by phosphorylation and arrestin binding.

Over 100 rhodopsin mutations have been linked to $\sim 30 \%$ of patients diagnosed with autosomal dominant retinitis pigmentosa (ADRP), a blinding disorder that affects one in 3000 people (RetNet, http://www.sph.uth.tmc.edu/RetNet/home.htm). Functional studies of some rhodopsin mutants in ADRP have led to their classification into distinct groups that implicate misfolding, mistrafficking, and constitutive activity as underlying bases for pho-

Received July 26, 2006; revised 0ct. 5, 2006; accepted 0ct. 6, 2006.

This work was supported by National Institutes of Health Grant EY12155 and the Arnold and Mabel Beckman Macular Research Center. We express our gratitude to Dr. Juan Korenbrot for his help assembling our electroretinogram apparatus and in the experimental design and data analysis pertaining to ERG recordings. We thank Dr. Tiansen Li for kindly providing the K296E transgenic mice and Dr. Paul Hargrave for generous gifts of the rhodopsin antibodies A11-82P and R2-12N.

Correspondence should be addressed to Jeannie Chen, Zilkha Neurogenetic Institute, Keck School of Medicine, University of Southern California, 1501 San Pablo Street, Room 223, Los Angeles, CA 90033. E-mail: jeannie@usc.edu.

G. Shi's present address: Pathology Department, Edwards Building, Room 301, Stanford University Medical Center, 300 Pasteur Drive, Stanford, CA 94305.

DOl:10.1523/JNEUROSCI.3212-06.2006

Copyright $\odot 2006$ Society for Neuroscience $\quad$ 0270-6474/06/2611929-09\$15.00/0 toreceptor cell death (Sung et al., 1993; Kaushal and Khorana, 1994; DeCaluwe and DeGrip, 1996; Deretic et al., 1998; Mendes et al., 2005). Yet, a functionally distinct group of mutations is suggested by the Drosophila visual system, wherein light-dependent formation of stable rhodopsin/arrestin complex was implicated in photoreceptor cell death (Alloway et al., 2000; Kiselev et al., 2000; Iakhine et al., 2004). In Drosophila, preventing the formation of rhodopsin/ arrestin complex circumvents the degeneration phenotype. Whether this pathway is conserved in the vertebrate retina is not known and cannot be assumed in view of substantial differences that exist between animals in these two phyla with respect to the lightinduced modes of cell death, the arrangement of cellular compartments, and the phototransduction signaling pathways. Nevertheless, some indirect evidence suggests that rhodopsin/arrestin complex may be a pathogenic mechanism for certain ADRP in human patients as well. For example, disruption of opsin transport by conditional knock-out of kinesin-II subunit KIF3A in mouse photoreceptor cells leads to accumulation of both opsin and arrestin in the inner segment compartment of rod cells before cell death (Marszalek et al., 2000). In addition, certain mutations that affect the R135 residue of opsin lead to formation of opsin/arrestin complex and disrupted receptor-mediated endocytic functions in a cell culture system (Chuang et al., 2004). Another opsin mutant, K296E, was thought to cause ADRP by constitutive stimulation of phototransduction, a notion supported by an in vitro finding that recombinantly expressed K296E catalyzed GTP loading in transducin in a light-independent manner (Robinson et al., 1992). However, in a transgenic mouse model, K296E was phosphorylated and bound with arrestin in the 
rod photoreceptor cells, and purified outer segments from these mice did not activate transducin in vitro until the samples were stripped of arrestin and dephosphorylated (Li et al., 1995). Thus, the basis for photoreceptor cell death mediated by K296E had heretofore remained unknown.

Testing for a toxic effect of rhodopsin/ arrestin complex in the vertebrate visual system requires an experimental paradigm that allows us to manipulate and control the formation of such complexes and to observe its effect on retinal degeneration. To this end, we used transgenic mice expressing the K296E opsin mutation. Formation of the rhodopsin/arrestin complex can be prevented by breeding the K296E transgene into the arr $1-/-$ background (supplemental Fig. 1, available at www. jneurosci.org as supplemental material). However, in the absence of arrestin, the constitutive activity of K296E may become unmasked, thereby promoting photoreceptor cell death through another mechanism: persistent stimulation of the phototransduction cascade (Fain and Lisman, 1993; J. Chen et al., 1999; Fain and Lisman, 1999; Fain, 2006). Therefore, the K296E $\mathrm{E}^{\text {arr1-/- }}$ mice were further crossed into the rod transducin $\alpha$ subunit knockout $(\mathrm{Tr}-/-$ ) background (Calvert et al., 2000) (supplemental Fig. 1, available at www.jneurosci.org as supplemental material). In this study, we showed that the retinal morphology of $\mathrm{K}_{2} 96 \mathrm{E}^{\mathrm{arr} 1-/-\mathrm{Tr}-/-}$ mice improved significantly compared with age-matched K296E mice. Furthermore, we provide evidence that $\mathrm{K} 296 \mathrm{E}$ is hyperphosphorylated and have discovered that its interaction with arrestin in the inner segment causes its mislocalization to that cellular compartment. These results provide direct evidence that rhodopsin/arrestin complex is toxic to vertebrate rod photoreceptor cells. Moreover, they suggest that mislocalization of rhodopsin/arrestin complex to the inner segment plays a role in initiating the signaling pathway that leads to cell death.

\section{Materials and Methods}

\section{Generation of mouse lines}

All experimental procedures were performed in accordance with regulations established by the National Institutes of Health, as well as with the Society for Neuroscience Policy on Animal Use in Neuroscience Research. K296E transgenic mice that expressed human K296E mutant opsin were obtained from Dr. Tiansen Li (Li et al., 1995). K296E transgenic mice were crossed with arr $1-/-, \operatorname{Tr}-/-$, and $\operatorname{arr} 1-/-\operatorname{Tr}-/-$ mice, respectively, to obtain $\mathrm{K}_{296 \mathrm{E}^{\text {arrl } 1-1-}, \mathrm{K}^{2} 96 \mathrm{E}^{\mathrm{Tr}-1-} \text {, and K296E }}^{\text {arr1-1-Tr-1-}}$ mice. K296E-negative littermates in the respective genetic backgrounds were used as age-matched controls. Unless clearly stated, all mice were born and raised in darkness to avoid light-dependent retinal degeneration (J. Chen et al., 1999; Hao et al., 2002). K296E was also crossed with rhodopsin-/- mice to increase the proportion of K296E protein in rod photoreceptor cells (Lem et al., 1999).

\section{Retinal morphometry}

Mice were killed under infrared light. The superior pole of the cornea was cauterized for orientation before enucleation. Eyecups were dissected, embedded into epoxy resin, and sectioned along the vertical meridian, as described previously (Concepcion et al., 2002). Thickness of the outer nuclear layer (ONL) was measured at 20 positions equally spaced along the retina (10 positions each in the superior and inferior hemispheres). For each position, three measurements were taken, and the average value of these three measurements was recorded. Measurements were made using a camera lucida connected to a light microscope, a WACOM graphics tablet (WACOM, Vancouver, WA), and AxioVision LE Rel. 4.1. software (Zeiss, Goettingen, Germany). Before each measurement session, the setup was calibrated using a stage micrometer (Klarmann Rulings, Litchfield, NH).

\section{Immunocytochemistry}

Dark-adapted 30-d-old K296E and K296E ${ }^{\text {arr1-1- }}$ mice were treated with $0.5 \%$ tropicamide and $2.5 \%$ phenylephrine hydrochloride to dilate the pupils before exposure to diffuse white light (2000 lux intensity) for 15 $\mathrm{min}$. Eyecups were then fixed, infiltrated with sucrose, and $10 \mu \mathrm{m}$ frozen 


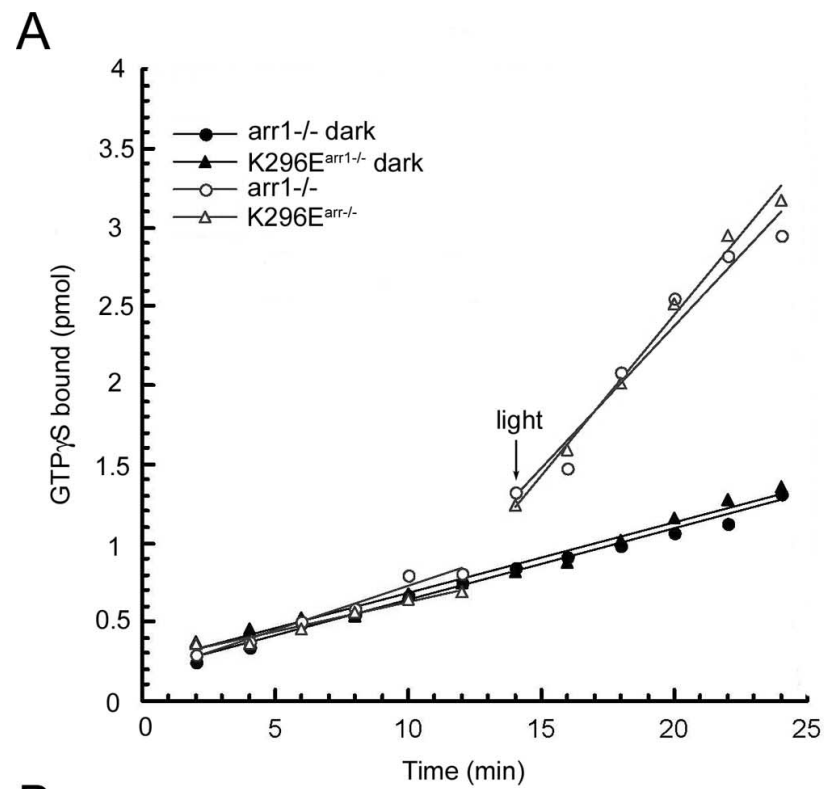

B

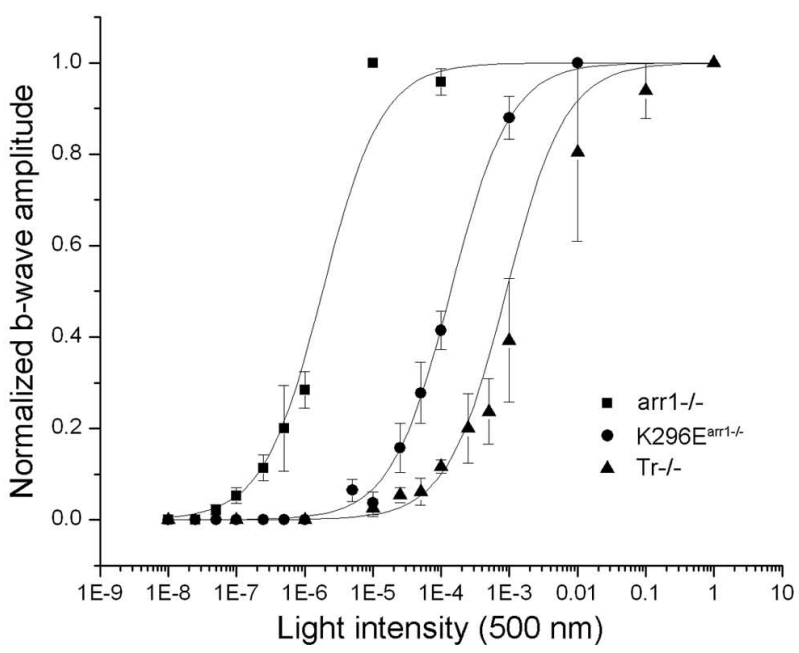

Figure 2. In vitro and in vivo analyses of K296E catalytic activity in the arr1-/- background. A, GTP $\gamma$ S loading assay catalyzed by rod outer segment preparations from arr1-/and K296E ${ }^{\text {arr1--1- }}$ retinas that contained $10 \mathrm{~nm}$ rhodopsin. Although catalytic activity of K296E was expected to result in an increased rate of GTP $\gamma 5$ loading in the dark-adapted samples, no such activity was observed. $B$, ERG recorded from 5 -week-old arr1-/- mice and their K296 $\mathrm{E}^{\text {arr1-1- }}$ littermates. Five-week-old $\mathrm{Tr}-1-$ mice were used to illustrate the cone threshold. Sensitivity was presented as normalized b-wave amplitude versus light intensity.

sections were obtained as described previously (Concepcion et al., 2002). Mouse monoclonal antibodies A11-82P and R2-12N (gifts from Dr. P. A. Hargrave, University of Florida, Gainesville, FL) recognized phosphorylated opsin and the $\mathrm{N}$ terminus of opsin, respectively. A rabbit polyclonal antibody raised against the $\mathrm{C} 10 \mathrm{C} 10$ epitope was used to visualize rod arrestin (Mendez et al., 2003). Images were acquired on an Axioplan2 microscope (Zeiss). All images for each section were taken at the same detection gain.

\section{Western blot analysis}

K296E phosphorylation assay. Thirty-day-old K296E transgenic mice and their transgene-negative littermates were dark-adapted overnight. Retinas were dissected under infrared light and exposed to 515-650 nm of light from a $100 \mathrm{~W}$ quartz tungsten halogen lamp (Oriel Instruments, Stratford, CT). The number of photons delivered was measured using a calibrated photodiode (United Detector Technology Sensors, Hawthorne, CA), and light delivery was controlled by neutral density filters
(Oriel Instruments) and an electromagnetic shutter (Vincent Associates, Rochester, NY). Retinas were exposed to light that caused 1, 5, 10, and $20 \%$ bleach or kept in the dark. Rhodopsin phosphorylation was allowed to proceed for an additional $10 \mathrm{~min}$ in the dark before the reaction was terminated by freezing in liquid $\mathrm{N}_{2}$. Frozen retinas were then homogenized in $100 \mu \mathrm{l}$ buffer ( $80 \mathrm{~mm}$ Tris, $\mathrm{pH} 8.0,4 \mathrm{~mm} \mathrm{MgCl}_{2}$ ) containing protease inhibitor mixture at 1 tablet per $10 \mathrm{ml}$ buffer (Roche Diagnostics, Indianapolis, IN). DNase I (30 U; Roche Diagnostics) was added and incubated at room temperature for $30 \mathrm{~min}$. An equal amount of retinal homogenate from each sample was loaded onto a $12 \%$ Bis-Tris SDSPAGE gel (Invitrogen., Carlsbad, CA). Proteins were transferred onto nitrocellulose membrane and incubated with A11-82P or R2-12N and visualized using enhanced chemiluminescence (GE Healthcare, Piscataway, NJ).

Rhodopsin/arrestin complex stability assay. The samples were prepared following the procedures described previously (Li et al., 1995) and blotted with rabbit polyclonal arrestin antibody raised against the $\mathrm{C} 10 \mathrm{C} 10$ epitope.

\section{GTP $\gamma$ S assay}

Retinas from arr1-/- or K296 $\mathrm{E}^{\text {arr1-/- }}$ mice were dissected under infrared light. Ten retinas were pooled per tube, and outer segments were isolated as described previously (Tsang et al., 1998). Rhodopsin was quantified by the Pierce (Rockford, IL) BCA assay kit. Transducin GTP $\gamma \mathrm{S}$ loading assays were performed as described previously (Robinson et al., 1992).

\section{Electroretinogram}

Mice were dark-adapted overnight $(>12 \mathrm{~h})$ and processed under infrared light. The mice were anesthetized with an intraperitoneal injection of xylazine $(10 \mu \mathrm{g} / \mathrm{g}$ body weight) and ketamine (100 $\mu \mathrm{g} / \mathrm{g}$ body weight) mixture. Pupils were dilated with $0.5 \%$ tropicamide and $2.5 \%$ phenylephrine hydrochloride. A drop of hydroxypropyl methylcellulose solution (Akorn, Buffalo Grove, IL) was placed on the cornea to keep the eye moist and to establish electrical contact with the corneal electrode. A steel needle reference electrode was placed subcutaneously below the eye. The flash intensity and its spectral composition were controlled with neutral density and narrow bandpass interference filters ( $500 \pm 10 \mathrm{~nm}$ full-width half-maximum). Electroretinogram (ERG) signals were amplified by an AC/DC differential amplifier (A-M Systems, Carlsborg, WA), bandpassfiltered at $0.1-1000 \mathrm{~Hz}$, sampled at $2000 \mathrm{~Hz}$, and acquired with a Digidata 1322A data acquisition board (Molecular Devices, Union City, CA) using pClamp software (Molecular Devices ). Sensitivity measurements were based on normalized b-wave amplitudes. To smooth the b-wave traces, a Gaussian filter with a bandwidth of $16.6 \mathrm{~Hz}$ at $3 \mathrm{~dB}$ cutoff was applied as described previously (Lyubarsky et al., 1999).

\section{Sample preparation for liquid chromatography mass spectrometry analysis}

Sample preparation and mass spectrometry analysis of rhodopsin phosphorylation were performed as described previously (Kennedy et al., 2001; Shi et al., 2005). Synthetic C-terminal peptides corresponding to mouse and human rhodopsin were used to determine their elution profile and ionization efficiency. Phosphates were removed from rhodopsin by calf intestine phosphatase treatment of retinal membranes from $\mathrm{K}_{296 \mathrm{E}^{\text {arr 1-1- }}}$ and K296E mice before LC-MS analysis.

\section{Results}

K296E transgenic mice were crossed into arr $1-/-$, $\mathrm{Tr}-/-$, and arr $1-/-\operatorname{Tr}-/-$ backgrounds to test the hypothesis that persistent rhodopsin/arrestin complex is toxic to vertebrate photoreceptor cells. Unless stated otherwise, all mice were born and raised in darkness to eliminate the possible effect of light exposure on retinal morphology. All mice were in a pigmented background. Dark-reared K296E-negative control mice (arr1-/-, $\operatorname{Tr}-/-$, and arr $1-/-\operatorname{Tr}-/-$ ) exhibited retinal morphology that was indistinguishable from wild-type mice during the time course of our study (Fig. 1A,E, I, M). Thus, the absence of arres- 
tin, transducin, or both had no discernible impact on retinal morphology.

\section{K296E-induced retinal degeneration is transducin independent}

The ratio of K296E transcript to endogenous opsin mRNA had been established previously as $0.25: 1$ in the line of transgenic mice used in this study (K296E-A) (Li et al., 1995). This low expression level precludes overexpression of opsin as an underlying cause of photoreceptor cell death (Olsson et al., 1992; Li et al., 1996). Consistent with the previous report ( $\mathrm{Li}$ et al., 1995), the K296E-A transgenic mice showed a slow time course of progressive light-independent retinal degeneration (Fig. $1 B-D$ ). Thinning of the ONL resulting from death of photoreceptor cells was apparent at 8 weeks of age. At 10 weeks, the ONL was reduced by $\sim 40 \%$ compared with the transgene-negative littermates. Slight disorganization of the outer segment structure was also apparent, but the overall length of the outer segment was not altered. The rate of degeneration increased when K296E was expressed in the rhodopsin +/ - background, which lends additional support for the causative role of K296E, rather than opsin overexpression, in promoting photoreceptor cell death (supplemental Fig. 2, available at www.jneurosci.org as supplemental material).

The time course of retinal degeneration and the appearance of retinal morphology in $\mathrm{K}_{2} 96 \mathrm{E}^{\mathrm{Tr}-1-}$ mice was indistinguishable from K296E $\mathrm{E}^{\mathrm{Tr}+/+}$ (Fig. $1 \mathrm{~F}-H$ ). These data confirm the conclusion reached by Li et al. (1995) that the K296E mutation does not cause retinal degeneration through constitutive activation of phototransduction. Rather, the data are consistent with the current hypothesis that formation of $\mathrm{K} 296 \mathrm{E} /$ arrestin complex is the underlying basis for photoreceptor cell death in this transgenic mouse model.

K296E activates transducin and causes retinal degeneration in the arr 1-/- background

K296E transgenic mice were crossed into the arr1-/- background to eliminate the formation of K296E/arrestin complex. As predicted, however, retinal degeneration was also observed in K296E $\mathrm{E}^{\text {arr1-l- }}$ mice (Fig. 1J-L). Interestingly, although the rate of degeneration was similar to that of K296E and $\mathrm{K} 296 \mathrm{E}^{\mathrm{Tr}-1-}$ mice, K296E $\mathrm{E}^{\text {arr 1-1- }}$ retinas showed a different degeneration pattern (i.e., the outer segments were noticeably shorter and more disorganized) and resembled the morphology of light-damaged retinas of albino rats or pigmented arr $1-/-$ mice exposed to low levels of light (Schremser and Williams, 1995; J. Chen et al., 1999), suggesting that K296E activated transducin in the arrestin knock-out background. To test this notion, we sought to determine whether outer segment preparations from K296E $\mathrm{E}^{\text {arr 1-I- }}$ mice exhibited light-independent GTP $\gamma \mathrm{S}$ loading of transducin in vitro. Figure $2 A$ shows transducin activation by rod outer segment membranes isolated from arr1-/- and K296E $\mathrm{E}^{\text {arr1- I- }}$ mice. As expected, both samples activated transducin in a lightdependent manner resulting from the presence of endogenous rhodopsin. However, no difference was seen between arr1-/- and K296E $\mathrm{E}^{\text {arr1-/- }}$ samples in the dark. Notably, Li et al. (1995) observed light-independent GTP $\gamma S$ loading of transducin by K296E only after removal of arrestin and phosphatase treatment. Thus, it is likely that phosphorylation greatly reduced the constitutive activity of K296E (see also below).

We then used the ERG as an alternative method to detect light-independent activity of K296E in the arr1-/- retina. If K296E activated transducin in the arr 1-/- background, then retinas from dark-reared $\mathrm{K}_{2} 96 \mathrm{E}^{\text {arr1-1- }}$ mice should have behaved as if light adapted and would have exhibited an elevated light threshold. To determine whether this was the case, ERG responses were compared between dark-adapted K296E arr1-/mice and K296E-negative arr1-/ - littermate controls. $\mathrm{Tr}-/-$ mice were also included in the comparison to demarcate the cone threshold. Figure $2 B$ shows that K296E $\mathrm{E}^{\text {arr1-1- }}$ mice displayed a $\sim 100$-fold decrease in flash sensitivity when compared with arr $1-/-$ mice. However, this lowered sensitivity was still one log unit above the cone threshold, indicating that $\mathrm{K} 296 \mathrm{E}^{\text {arr1-/- }}$ rods were not saturated. These results support the notion that, in the absence of arrestin, K296E constitutively activated transducin, thereby acting as a source of "dark" light to cause photoreceptor cell death in K296E $\mathrm{E}^{\text {arrl-1- }}$ mice.

K296E-induced retinal degeneration is slowed in the absence of arrestin and transducin

K296E $\mathrm{E}^{\text {arr 1-I- }}$ mice were further crossed into the $\mathrm{Tr}-/-$ background to prevent retinal degeneration induced by persistent stimulation of phototransduction. Remarkably, K296E $\mathrm{E}^{\text {arr } 1-1-\mathrm{Tr}-1-}$ mice exhibited substantially improved retinal morphology when compared with age-matched K296E, K296 $\mathrm{E}^{\text {arr1-l- }}$, or K296 $\mathrm{E}^{\mathrm{Tr}-1-}$ mice. The number of photoreceptor cells, as reflected by the thickness of the ONL, was similar to that of the transgene-negative controls, as was the length and the organized structure of the outer segment (Fig. $1 N-P$ ). To better quantify the degree of rescue, ONL thickness was measured at 20 positions, spaced apart equally, that spanned the vertical meridian of the eye. As can be seen in Figure 3, 
the ONL thickness of $\mathrm{K} 296 \mathrm{E}^{\mathrm{arr} 1-/-\mathrm{Tr}-1-}$ retinas was similar to that of the transgene-negative controls at the periphery of the retina, but the ONL was noticeably thinner at the central region of the retina. Nevertheless, the arr $1-/-\operatorname{Tr}-/-$ background provided a higher degree of rescue from K296E-induced retinal degeneration when compared with K296E in the single knock-out backgrounds (Figs. $1 d, 3)$. These results are consistent with the hypothesis that accumulation of stable rhodopsin/arrestin complex is indeed toxic to mammalian photoreceptor cells. However, preventing this complex formation was not sufficient to completely rescue K296E-induced retinal degeneration.

Constitutive activity of $\mathrm{K} 296 \mathrm{E}$ causes retinal degeneration in arr $1-/-\mathrm{Tr}-/-$ retinas

We further explored the possibility that the constitutive activity of K296E is the underlying cause of photoreceptor cell death observed in dark-reared $\mathrm{K} 296 \mathrm{E}^{\mathrm{arrl}-1-\mathrm{Tr}-1-}$ mice. To test this hypothesis, arr $1-/-\mathrm{Tr}-/-$ mice were exposed to constant light for 1 week to examine whether light-activated rhodopsin $\left(\mathrm{R}^{\star}\right)$ had an effect similar to K296E on the retinal morphology of arr $1-/-\operatorname{Tr}-/-$ mice. As can be seen in Figure 4, the central region of the light-exposed arr $1-/-\operatorname{Tr}-/-$ retina was severely damaged. This pattern of light damage to the central retina closely mirrored that seen in $\mathrm{K} 296 \mathrm{E}^{\mathrm{arr} 1-/-\mathrm{Tr}-1-}$ retinas and was unlike the pattern seen in other rodent models of light damage where sensitivity to light damage was exhibited by either the superior or the inferior region of the retina (LaVail et al., 1987; J. Chen et al., 1999; Hao et al., 2002; Roca et al., 2004). Thus, $\mathrm{R}^{*}$, as well as $\mathrm{K} 296 \mathrm{E}$, is able to signal through a transducin- and arrestin-independent pathway to induce retinal degeneration.

\section{$\mathrm{K} 296 \mathrm{E}$ is colocalized with arrestin in the retina}

Immunocytochemistry was performed to verify that K296E colocalizes with arrestin (Fig. 5). Rhodopsin, in the active conformation, is a substrate for rhodopsin kinase, which places multiple phosphate groups at a cluster of Ser and Thr sites at the C terminus of rhodopsin (Wilden and Kuhn, 1982; Palczewski et al., 1988; Palczewski and Benovic, 1991). Activated, phosphorylated rhodopsin is then capped by arrestin binding (Wilden et al., 1986; $\mathrm{Xu}$ et al., 1997). Thus, arrestin is expected to colocalize with $\mathrm{R}^{\star}$ in a light-dependent manner and K296E in a light-independent manner, because $\mathrm{K} 296 \mathrm{E}$ is in an active conformation regardless of light. A monoclonal antibody, A11-82P, binds strongly to multiply phosphorylated rhodopsin but not to unphosphorylated rhodopsin (Adamus et al., 1988). As shown in Figure 5, A11-82P reactivity was observed only in the rod outer segment of lightexposed wild-type retina where phosphorylated $\mathrm{R}^{\star}$ is localized. In contrast, A11-82P reactivity was observed in both the darkadapted and light-adapted retinas of K296E mice, indicating that $\mathrm{K} 296 \mathrm{E}$ is constitutively phosphorylated. It is also noteworthy that the A11-82P staining pattern in the wild-type retina is restricted to the outer segment where rhodopsin is localized, whereas in the K296E retina, the A11-82P reactivity is seen throughout the photoreceptor layer, indicating mislocalization of phosphorylated K296E. Thus, K296E, being in an active conformation, is a substrate for rhodopsin kinase in all compartments of the rod photoreceptor cell, even in the absence of light.

Arrestin is known to exhibit a light-dependent distribution pattern in the retina (Broekhuyse et al., 1985, 1987; Mangini and Pepperberg, 1988; Whelan and McGinnis, 1988; Peterson et al., 2003). In the dark, arrestin is primarily excluded from the outer segment compartment and resides primarily in the inner segment and ONL (Fig. 5B). After light exposure, arrestin moves to the

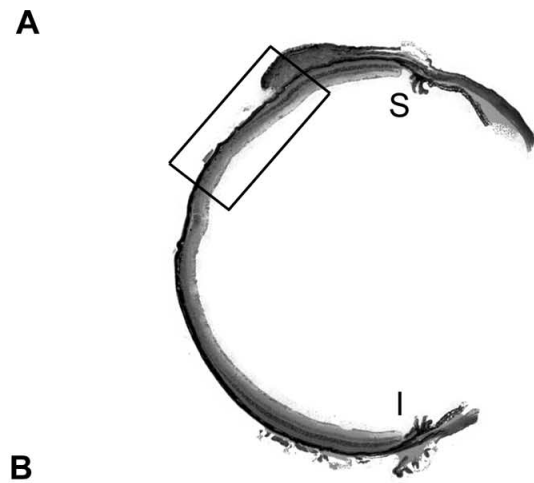

B
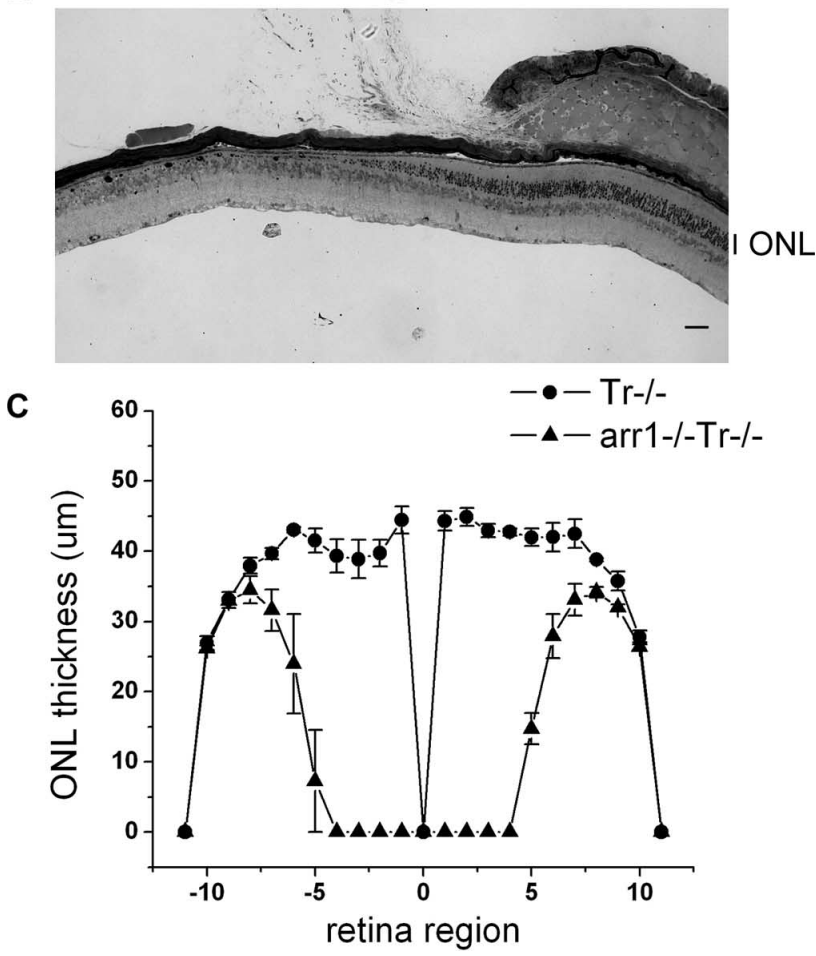

Figure 4. Pattern of retinal degeneration in dark-reared $\mathrm{K} 296 \mathrm{E}^{\text {arr1-l- } \mathrm{Tr}-1-}$ mice is recapitulated in light exposure of arr1 $-/-\mathrm{Tr}-/-$ mice. Representative retinal sections obtained from arr1 $-/-\mathrm{Tr}-/-$ mice (pupils undilated) were exposed to constant illumination of 2000 lux for 1 week. $\boldsymbol{A}$, Low-magnification view of the whole retina. $\boldsymbol{B}$, Higher magnification of the boxed area shown in $\boldsymbol{A}$. $\boldsymbol{C}$, Morphometric measurements of the outer nuclear layer thickness $(n=3)$. Error bars represent SD. S, Superior $(-10)$; I, inferior (10). Scale bar, $50 \mu \mathrm{m}$.

outer segment (Fig. 5E). This light-dependent distribution of arrestin was not seen in the $\mathrm{K} 296 \mathrm{E}$ retina. Regardless of lighting conditions, arrestin appeared to be distributed throughout the photoreceptor cell layer, mirroring the pattern of phosphorylated K296E (Fig. 5H,K). The colocalization of arrestin with phosphorylated K296E is consistent with the notion that they exist as a complex in the rod photoreceptor cell.

\section{$\mathrm{K} 296 \mathrm{E}$ is retained in the inner segment and ONL through its interaction with arrestin}

We explored the cause of K296E mislocalization to the inner segment and the ONL. It is known that the C-terminal domain of rhodopsin contains a trafficking signal that is necessary and sufficient to direct polarized transport of rhodopsin to the outer segment (Sung et al., 1994; Deretic, 1998; Deretic et al., 1998; Tam et al., 2000; Concepcion et al., 2002). Immediately preceding this domain is a cluster of Ser and Thr residues that, after phosphorylation, participate in converting arrestin from its latent, 
inactive state into a conformation that binds light-activated, phosphorylated rhodopsin with high affinity (Hirsch et al., 1999; Vishnivetskiy et al., 2000; Gurevich and Gurevich, 2004). Therefore, it is plausible that the binding of arrestin may have masked the C-terminal domain of K296E that is normally required for rhodopsin to traffic to the outer segment. Another possibility is that a proportion of $\mathrm{K} 296 \mathrm{E}$ is misfolded, because misfolded rhodopsin is also retained in the inner segment and becomes mislocalized (Olsson et al., 1992) and causes cell death through protein aggregation and disruption of protein degradation (Kopito, 2000; Rajan et al., 2001; Illing et al., 2002). To distinguish between these two possibilities, localization of rhodopsin and K296E was investigated in K296E $\mathrm{E}^{\text {arr1-/- }}$ retinas using an antibody against the $\mathrm{N}$ terminus of rhodopsin, R2$12 \mathrm{~N}$ (Fig. 6). In the wild-type retina, rhodopsin is localized predominantly to the membranous outer segment. In the K296E retinas, however, rhodopsin immunoreactivity was seen throughout the photoreceptor cell layer, a result similar to that obtained with the anti-phosphorylated opsin antibody, A11-82P (Fig. 6B). Remarkably, in the absence of arrestin, K296E mutant opsin reactivity was shifted to the outer segment (Fig. 6D). These results suggest that $\mathrm{K} 296 \mathrm{E}$ is correctly folded but is retained in the inner segment and ONL through its interaction with arrestin.

\section{Hyperphosphorylation of $\mathrm{K} 296 \mathrm{E}$ may contribute to stable complex formation with arrestin}

The results shown in Figure $2 B$ indicate that the rod responses from K296 $\mathrm{E}^{\text {arr1-/- }}$ mice were not saturated. This result is somewhat surprising, because the level of K296E transcript was estimated to be $25 \%$ of total rhodopsin transcripts (Li et al., 1995). This level of K296E protein expression would be equivalent to a steady-state light exposure that bleached $25 \%$ of rhodopsin at any given time. However, it is known that in primate and mouse rods, saturation is reached after $\sim 400 \mathrm{R}^{*} \cdot \mathrm{s}{ }^{-1}$ to $\sim 4000 \mathrm{R}^{*} \cdot \mathrm{s}{ }^{-1}$, or $\sim 0.001-0.01 \%$ bleach $\cdot{ }^{-1}$, respectively, under steady background light (Baylor et al., 1984; Mendez et al., 2001; Makino et al., 2004), which is a value substantially lower than $25 \%$ bleach $\cdot \mathrm{s}^{-1}$. We quantified the level of K296E protein using a mass spectrometry-based method (Kennedy et al., 2001; Shi et al., 2005) to directly assess the level of K296E protein. Shown in supplemental Figure 3 (available at www.jneurosci.org as supplemental material) are representative ion chromatograms of C-terminal peptides that correspond to mouse rhodopsin and K296E released by endopeptidase Asp-N treatment of K296 $\mathrm{E}^{\mathrm{RK}-1-}$ retinal membranes. K296 $\mathrm{E}^{\mathrm{RK}-1-}$ retina was used to ensure that neither K296E nor rhodopsin would be phosphorylated, thus simplifying quantification. Using this LC-MS method, we estimated the proportion of $\mathrm{K} 296 \mathrm{E}$ to endogenous mouse rhodopsin in the following genetic backgrounds (mean $\pm \mathrm{SD}$; $n=3$ for each condition): $\mathrm{K}_{2} 96 \mathrm{E}^{\mathrm{RK}-/-}, 1.04 \% \pm 0.06 \%$; $\mathrm{K} 296 \mathrm{E}^{\mathrm{arr} 1-/-}, 1.33 \% \pm 0.03 \%$; K296E, $4.5 \% \pm 0.8 \%$. Phos- phates were removed by phosphatase treatment in the latter two samples before LC-MS analysis. The discrepancy between the values obtained from K296E, K296 $\mathrm{E}^{\text {arr1-l- }}$, and K296E $\mathrm{E}^{\mathrm{RK}-1-}$ samples may reflect the shortened outer segments observed when K296E is expressed in the arr1-/- or RK-/- genetic backgrounds. These results indicate that the proportion of the K296E protein to endogenous mouse rhodopsin was much lower than the value obtained by transcript analysis.

A comparison between single-photon responses obtained from RK $-/-$ rods (C. K. Chen et al., 1999) and arr1-/- rods (Xu et al., 1997) indicates that phosphorylation alone decreases the catalytic activity of $\mathrm{R}^{\star}$ by $\sim 70 \%$. Thus, an expression level of $1 \% \mathrm{~K} 296 \mathrm{E}$ protein in the arr $1-/-$ background is still expected to exceed the rod saturation threshold of $\sim 0.001 \%$ bleach $^{-1}{ }^{-1}$, even if phosphorylation decreased the catalytic activity of K296E. However, it is known that heavily phosphorylated rhodopsin exhibits greatly reduced catalytic activity in vitro (Arshavsky et al., 1985; Wilden, 1995). For this reason, we used the A11-82P antibody, which preferentially binds highly phosphorylated species of rhodopsin (Adamus et al., 1988), to compare the extent of phosphorylation in $\mathrm{K} 296 \mathrm{E}$ and in rhodopsin exposed to calibrated light that produced $1,5,10$, and $20 \%$ bleach. As can be seen in Figure $7 A$, the signal from retinas that expressed $4.5 \%$ K296E exceeded that of control retinas exposed to $20 \%$ bleach, suggesting that $\mathrm{K} 296 \mathrm{E}$ is phosphorylated to a greater extent than is wild-type rhodopsin. Thus, hyperphosphorylation is likely the reason for our inability to detect K296E-catalyzed GTP- $\gamma \mathrm{S}$ loading of transducin in vitro (Fig. $2 \mathrm{~A}$ ) and its inability to saturate rod 

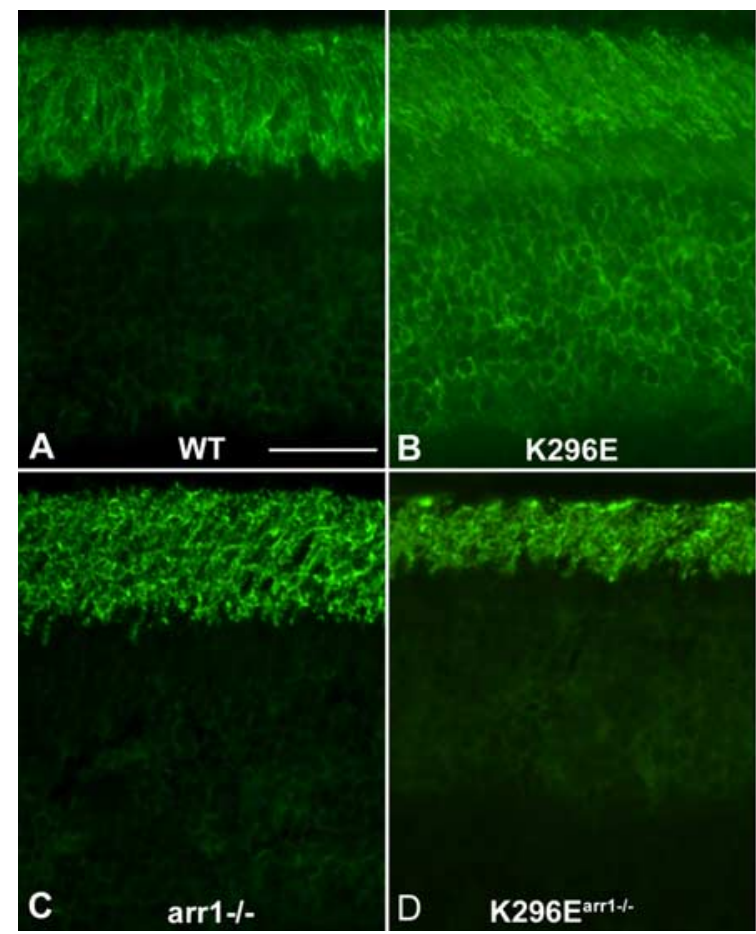

OS

IS

ONL
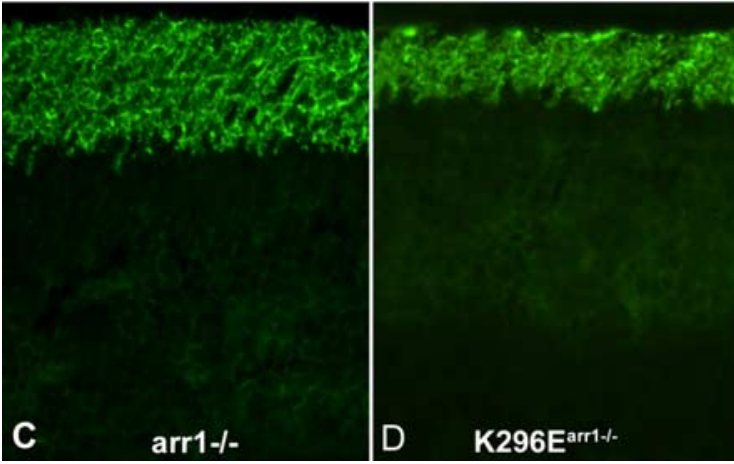

Figure 6. K296E was retained in the inner segment and ONL via its interaction with arrestin Rhodopsin was visualized using R2-12N, a mouse monoclonal antibody that recognizes the $\mathrm{N}$-terminal 2-12 residues of rhodopsin (Adamus et al., 1991). Rhodopsin localized to the outer segment in wild-type (WT) and arr1-/- retinas $(\boldsymbol{A}, \boldsymbol{C})$. In the retina expressing K296E, rhodopsin reactivity was seen throughout the retinal layers $(\boldsymbol{B})$. In the $\mathrm{K} 296 \mathrm{E}^{\text {arr1- }-1-}$ retina, all rhodopsin reactivity was seen in the outer segment (D). OS, Outer segment; IS, inner segment. Scale bar, $25 \mu \mathrm{m}$.

response in arr $1-/-$ mice (Fig. $2 B$ ). To compare the relative stability of K296E/arrestin complex to $\mathrm{R}^{\star} /$ arrestin complex, pellet fractions from retinal homogenates were subjected to sequential, increasingly stringent washes (Fig. $7 B$ ). Similar amounts of arrestin were present in the original pellet fractions of dark- or light-adapted retinas (Fig. $7 B$, unwashed). After a hypotonic wash, the majority of arrestin was removed from the darkadapted wild-type sample but not from the K296E sample, whereas in the light-adapted samples, comparable amounts of arrestin were retained. After the more stringent urea wash, the remaining bound arrestin was removed from the light-treated wild-type sample but not from the K296E sample. Thus, the K296E/arrestin complex appears to be more stable than the $\mathrm{R}^{\star}$ I arrestin complex formed as a consequence of normal light exposure. This increased stability may be attributed to the observed hyperphosphorylation of K296E.

\section{Discussion}

The amelioration of K296E-induced retinal degeneration in the arrestin/transducin double knock-out provide direct evidence that persistent rhodopsin/arrestin complex is a stimulus for photoreceptor cell death in the vertebrate visual system. Thus, this retinal degeneration pathway appears to be conserved from the invertebrate to the vertebrate visual system. In Drosophila, endocytosis of rhodopsin/arrestin complex is a required step in this signaling pathway. Additional investigation is necessary to determine whether endocytosis is a required step leading to cell death in the vertebrate retina.

It is important to note that certain naturally occurring mutations affecting the $\mathrm{C}$ terminus of rhodopsin, such as Q344ter and

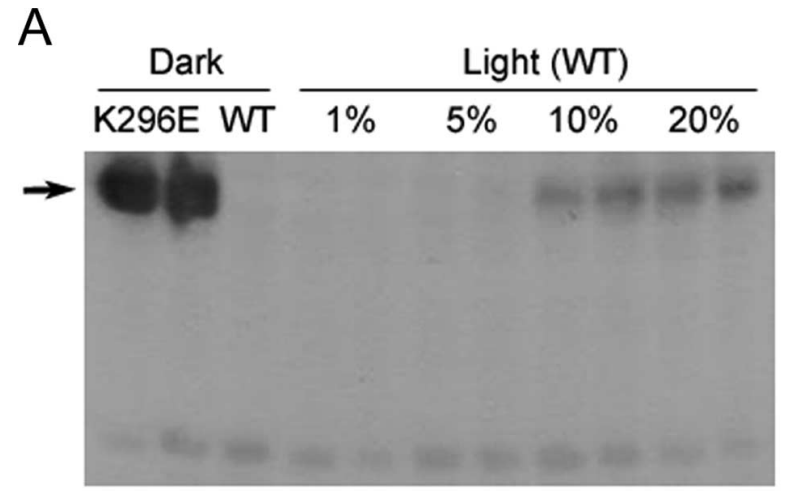

B

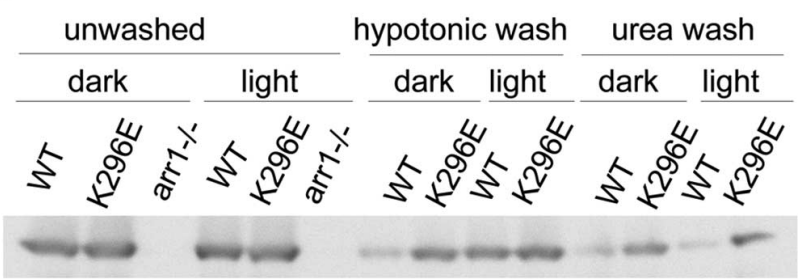

Figure 7. K296E was highly phosphorylated and formed a stable complex with arrestin. $\boldsymbol{A}$, Retinal homogenates from dark-adapted $\mathrm{K} 296 \mathrm{E}$ retina and retinas isolated from wild-type (WT) mice exposed to calibrated light that produced an estimated $0,1,5,10$, and 20\% bleach. Highly phosphorylated opsin was detected by A11-82P antibody. Remarkably, K296E samples, with an estimated expression level of $4.5 \pm 0.8 \%$, showed a much stronger signal than did wild-type retinas exposed to $20 \%$ bleach. $B$, Membrane fraction from retinal homogenates obtained from dark-adapted or light-exposed mice was subjected to hypotonic wash followed by urea wash. The most stringent urea wash stripped most of the arrestin from the light-exposed retina of the wild-type sample but not from the K296E sample.

P347S, perturb rhodopsin trafficking to the outer segment. The ensuing mislocalization is thought to be the underlying basis for ADRP in these instances (Sung et al., 1994; Deretic et al., 1996, 1998; Li et al., 1996). In a previous study to investigate the effect of rhodopsin mislocalization on retinal degeneration, we observed that mislocalization of a trafficking defective mutant, S334ter, did not lead to retinal degeneration in a transgenic mouse model when the protein was expressed at $10 \%$ of total rhodopsin levels (Concepcion et al., 2002). Thus it appears unlikely that K296E, when expressed at $4.5 \%$, exerted its toxicity through mislocalization alone.

Because arrestin binding to $\mathrm{R}^{*}$ is a step essential to rhodopsin deactivation, it can be expected that high levels of rhodopsin/ arrestin complex are formed in the rod outer segment during the course of bright light exposure. Unlike the Drosophila photoreceptors, the outer segment compartment of vertebrate rods is physically sequestered from the rest of the cell body via a thin connecting cilium and appears to lack space for endocytic machinery; indeed, endocytosis has not been observed in this compartment. How, then, does K296E/arrestin complex cause photoreceptor cell death? We speculate that the cause is the inappropriate accumulation of rhodopsin/arrestin complex in the inner segment compartments of the rod cell where endocytosis is supported. Importantly, we show that $\mathrm{K} 296 \mathrm{E}$ is retained in the proximal compartments, where it appears to form a stable complex with arrestin.

The 11-cis-retinal chromophore is covalently attached to K296 of the opsin protein through a protonated Schiff base linkage (Zhukovsky et al., 1991). Mutations affecting K296 render the opsin protein incapable of binding retinal and disrupt the inter- 
action between K296 and E113 that normally constrains the opsin protein to an inactive conformation (Robinson et al., 1992). Hence, K296E exists in an active conformation the moment it is synthesized in the inner segment and is phosphorylated and bound to arrestin in this compartment. The K296E/arrestin complex appears to be stable, because the ability of K296E to activate transducin is silenced by arrestin binding. It appears that hyperphosphorylation of K296E may be the underlying cause for this increased stability of K296E/arrestin complex as well as its greatly decreased ability to activate transducin in the arrestin knock-out background. Additional evidence in support of a stable complex derives from our observation that transphosphorylation of nonactivated rhodopsin was observed only when K296 was expressed in the arrestin knock-out background; when arrestin was present, K296E lost its ability to activate rhodopsin kinase to phosphorylate nearby, nonactivated rhodopsin molecules (Shi et al., 2005). In addition, ERG measurements showed no difference in sensitivity between wild-type mice and K296E mice (data not shown). These data support the notion that stable rhodopsin/arrestin complex accumulated in the inner segment initiates a transducin-independent signaling cascade that leads to photoreceptor cell death.

Formation of excessive rhodopsin/arrestin complex in the inner segment is likely to occur as a result of other mutations. For example, rhodopsin R135L mutants appear to be phosphorylated by rhodopsin kinase and bound to arrestin in vitro (Shi et al., 1998). When the R135L mutant is introduced into murine retina by in vivo electroporation, it appears to cause arrestin to accumulate around the cell body, suggesting that R135L/arrestin complex may form within these cellular compartments (Chuang et al., 2004). In addition, other naturally occurring mutations that affect the trafficking domain on the $\mathrm{C}$ terminus of rhodopsin will likely result in accumulation of rhodopsin in the inner segment and ONL (Sung et al., 1994; Li et al., 1996). Because these mutations often do not affect phosphorylation or arrestin binding, it is plausible that light activation of mislocalized mutant rhodopsin leads to excessive rhodopsin/arrestin complex in these cellular compartments. By the same token, defects in other components that affect normal rhodopsin transport to the outer segment (such as the aforementioned KIF3A mutation) will have a similar effect. Thus, excessive accumulation of rhodopsin/arrestin complex may define a broader class of mutations that lead to blindness.

Drosophila expresses two visual arrestins where Arr2 is more abundant and plays the major role in deactivating rhodopsin. Under normal light exposure, an increase in cytoplasmic $\mathrm{Ca}^{2+}$ concentration leads to Arr2 phosphorylation by $\mathrm{Ca}^{2+}$ / calmodulin-dependent protein kinase II (Kahn and Matsumoto, 1997). Phosphorylation of Arr2 destabilizes its binding to rhodopsin, thereby preventing accumulation of rhodopsin/Arr2 complex. In addition, Arrl appears to play an essential role in normal, light-induced rhodopsin endocytosis as well as in preventing accumulation of toxic rhodopsin/Arr2 complex; when Arr1 is absent, retinal degeneration ensues (Satoh and Ready, 2005). Thus, it appears that multiple mechanisms regulate the accumulation of rhodopsin/Arr2 complex formation during normal light exposure in the Drosophila photoreceptor cell. The mammalian rod photoreceptor cells express two isoforms of rod arrestin encoded by one gene: a $48 \mathrm{kDa}$ full-length arrestin and a less abundant splice variant that is truncated at the $\mathrm{C}$ terminus (Palczewski et al., 1994; Smith et al., 1994; Smith, 1996). Although the splice variant resembles Drosophila Arr1, it does not appear to play a part in mediating light-dependent rhodopsin endocytosis, because expression of the full-length protein alone in the arrestin knock-out mice had little effect on retinal morphology (Burns et al., 2006).

Retinal degeneration was not completely suppressed after placement of K296E in the arrestin/transducin double knock-out background. The residual degeneration appears to be caused by the activity of $\mathrm{K} 296 \mathrm{E}$, because light exposure recapitulated the same pattern of degeneration in arr $1-/-\mathrm{Tr}-/-$ retinas. Low light-induced retinal damage in pigmented mice caused by persistent phototransduction should have been blocked in the transducin knock-out background. However, a previous study showed that not all mice deficient in transducin are resistant to this mechanism of light damage, suggesting a genetic modifier effect (Hao et al., 2002). The nature of this signaling cascade leading to photoreceptor cell death is not yet known. It is possible that rhodopsin signaling through other G-proteins, such as Gi or Go, may be the underlying basis of this degeneration. Future studies will be required to fully understand the complex effect of light on retinal physiology.

\section{References}

Adamus G, Zam ZS, McDowell JH, Shaw GP, Hargrave PA (1988) A monoclonal antibody specific for the phosphorylated epitope of rhodopsin: comparison with other anti-phosphoprotein antibodies. Hybridoma 7:237-247.

Adamus G, Zam ZS, Arendt A, Palczewski K, McDowell JH, Hargrave PA (1991) Anti-rhodopsin monoclonal antibodies of defined specificity: characterization and application. Vision Res 31:17-31.

Alloway PG, Howard L, Dolph PJ (2000) The formation of stable rhodopsin-arrestin complexes induces apoptosis and photoreceptor cell degeneration. Neuron 28:129-138.

Arshavsky VY, Dizhoor AM, Shestakova IK, Philippov P (1985) The effect of rhodopsin phosphorylation on the light-dependent activation of phosphodiesterase from bovine rod outer segments. FEBS Lett 181:264-266.

Baylor DA, Nunn BJ, Schnapf JL (1984) The photocurrent, noise and spectral sensitivity of rods of the monkey Macaca fascicularis. J Physiol (Lond) 357:575-607.

Broekhuyse RM, Tolhuizen EF, Janssen AP, Winkens HJ (1985) Light induced shift and binding of S-antigen in retinal rods. Curr Eye Res 4:613-618.

Broekhuyse RM, Janssen AP, Tolhuizen EF (1987) Effect of light-adaptation on the binding of 48-kDa protein (S-antigen) to photoreceptor cell membranes. Curr Eye Res 6:607-610.

Burns ME, Mendez A, Chen CK, Almuete A, Quillinan N, Simon MI, Baylor DA, Chen J (2006) Deactivation of phosphorylated and nonphosphorylated rhodopsin by arrestin splice variants. J Neurosci 26:1036-1044.

Calvert PD, Krasnoperova NV, Lyubarsky AL, Isayama T, Nicolo M, Kosaras B, Wong G, Gannon KS, Margolskee RF, Sidman RL, Pugh Jr EN, Makino CL, Lem J (2000) Phototransduction in transgenic mice after targeted deletion of the rod transducin alpha -subunit. Proc Natl Acad Sci USA 97:13913-13918.

Chen CK, Burns ME, Spencer M, Niemi GA, Chen J, Hurley JB, Baylor DA, Simon MI (1999) Abnormal photoresponses and light-induced apoptosis in rods lacking rhodopsin kinase. Proc Natl Acad Sci USA 96:3718-3722.

Chen J, Simon MI, Matthes MT, Yasumura D, LaVail MM (1999) Increased susceptibility to light damage in an arrestin knockout mouse model of Oguchi disease (stationary night blindness). Invest Ophthalmol Vis Sci 40:2978-2982.

Chuang JZ, Vega C, Jun W, Sung CH (2004) Structural and functional impairment of endocytic pathways by retinitis pigmentosa mutant rhodopsin-arrestin complexes. J Clin Invest 114:131-140.

Concepcion F, Mendez A, Chen J (2002) The carboxyl-terminal domain is essential for rhodopsin transport in rod photoreceptors. Vision Res 42:417-426.

DeCaluwe GL, DeGrip WJ (1996) Point mutations in bovine opsin can be classified in four groups with respect to their effect on the biosynthetic pathway of opsin. Biochem J 320:807-815.

Deretic D (1998) Post-Golgi trafficking of rhodopsin in retinal photoreceptors. Eye 12:526-530.

Deretic D, Schmerl S, Hargrave PA, Arendt A, McDowell JH (1998) Regulation of sorting and post-Golgi trafficking of rhodopsin by its C-terminal sequence QVS(A)PA. Proc Natl Acad Sci USA 95:10620-10625. 
Fain GL, Lisman JE (1993) Photoreceptor degeneration in vitamin A deprivation and retinitis pigmentosa: the equivalent light hypothesis. Exp Eye Res 57:335-340.

Fain GL, Lisman JE (1999) Light, Ca2+, and photoreceptor death: new evidence for the equivalent-light hypothesis from arrestin knockout mice. Invest Ophthalmol Vis Sci 40:2770-2772.

Gurevich VV, Gurevich EV (2004) The molecular acrobatics of arrestin activation. Trends Pharmacol Sci 25:105-111.

Hao W, Wenzel A, Obin MS, Chen CK, Brill E, Krasnoperova NV, EversoleCire P, Kleyner Y, Taylor A, Simon MI, Grimm C, Reme CE, Lem J (2002) Evidence for two apoptotic pathways in light-induced retinal degeneration. Nat Genet 32:254-260.

Hirsch JA, Schubert C, Gurevich VV, Sigler PB (1999) The 2.8 A crystal structure of visual arrestin: a model for arrestin's regulation. Cell 97:257-269.

Iakhine R, Chorna-Ornan I, Zars T, Elia N, Cheng Y, Selinger Z, Minke B, Hyde DR (2004) Novel dominant rhodopsin mutation triggers two mechanisms of retinal degeneration and photoreceptor desensitization. J Neurosci 24:2516-2526.

Illing ME, Rajan RS, Bence NF, Kopito RR (2002) A rhodopsin mutant linked to autosomal dominant retinitis pigmentosa is prone to aggregate and interacts with the ubiquitin proteasome system. J Biol Chem 277:34150-34160.

Kahn ES, Matsumoto H (1997) Calcium/calmodulin-dependent kinase II phosphorylates Drosophila visual arrestin. J Neurochem 68:169-175.

Kaushal S, Khorana HG (1994) Structure and function in rhodopsin. 7. Point mutations associated with autosomal dominant retinitis pigmentosa. Biochemistry 33:6121-6128.

Kennedy MJ, Lee KA, Niemi GA, Craven KB, Garwin GG, Saari JC, Hurley JB (2001) Multiple phosphorylation of rhodopsin and the in vivo chemistry underlying rod photoreceptor dark adaptation. Neuron 31:87-101.

Kiselev A, Socolich M, Vinos J, Hardy RW, Zuker CS, Ranganathan R (2000) A molecular pathway for light-dependent photoreceptor apoptosis in Drosophila. Neuron 28:139-152.

Kopito RR (2000) Aggresomes, inclusion bodies and protein aggregation. Trends Cell Biol 10:524-530.

LaVail MM, Gorrin GM, Repaci MA, Yasumura D (1987) Light-induced retinal degeneration in albino mice and rats: strain and species differences. Prog Clin Biol Res 247:439-454.

Lem J, Krasnoperova NV, Calvert PD, Kosaras B, Cameron DA, Nicolo M, Makino CL, Sidman RL (1999) Morphological, physiological, and biochemical changes in rhodopsin knockout mice. Proc Natl Acad Sci USA 96:736-741.

Li T, Franson WK, Gordon JW, Berson EL, Dryja TP (1995) Constitutive activation of phototransduction by K296E opsin is not a cause of photoreceptor degeneration. Proc Natl Acad Sci USA 92:3551-3555.

Li T, Snyder WK, Olsson JE, Dryja TP (1996) Transgenic mice carrying the dominant rhodopsin mutation P347S: evidence for defective vectorial transport of rhodopsin to the outer segments. Proc Natl Acad Sci USA 93:14176-14181.

Lyubarsky AL, Falsini B, Pennesi ME, Valentini P, Pugh Jr EN (1999) UV- and midwave-sensitive cone-driven retinal responses of the mouse: a possible phenotype for coexpression of cone photopigments. J Neurosci 19:442-455.

Makino CL, Dodd RL, Chen J, Burns ME, Roca A, Simon MI, Baylor DA (2004) Recoverin regulates light-dependent phosphodiesterase activity in retinal rods. J Gen Physiol 123:729-741.

Mangini NJ, Pepperberg DR (1988) Immunolocalization of 48K in rod photoreceptors. Light and ATP increase OS labeling. Invest Ophthalmol Vis Sci 29:1221-1234.

Marszalek JR, Liu X, Roberts EA, Chui D, Marth JD, Williams DS, Goldstein LS (2000) Genetic evidence for selective transport of opsin and arrestin by kinesin-II in mammalian photoreceptors. Cell 102:175-187.

Mendes HF, van der Spuy J, Chapple JP, Cheetham ME (2005) Mechanisms of cell death in rhodopsin retinitis pigmentosa: implications for therapy. Trends Mol Med 11:177-185.

Mendez A, Burns ME, Sokal I, Dizhoor AM, Baehr W, Palczewski K, Baylor DA, Chen J (2001) Role of guanylate cyclase-activating proteins (GCAPs) in setting the flash sensitivity of rod photoreceptors. Proc Natl Acad Sci USA 98:9948-9953.

Mendez A, Lem J, Simon M, Chen J (2003) Light-dependent translocation of arrestin in the absence of rhodopsin phosphorylation and transducin signaling. J Neurosci 23:3124-3129.

Olsson JE, Gordon JW, Pawlyk BS, Roof D, Hayes A, Molday RS, Mukai S, Cowley GS, Berson EL, Dryja TP (1992) Transgenic mice with a rhodop- sin mutation (Pro23His): a mouse model of autosomal dominant retinitis pigmentosa. Neuron 9:815-830.

Palczewski K, Benovic JL (1991) G-protein-coupled receptor kinases. Trends Biochem Sci 16:387-391.

Palczewski K, McDowell JH, Hargrave PA (1988) Rhodopsin kinase: substrate specificity and factors that influence activity. Biochemistry 27:2306-2313.

Palczewski K, Buczylko J, Ohguro H, Annan RS, Carr SA, Crabb JW, Kaplan MW, Johnson RS, Walsh KA (1994) Characterization of a truncated form a arrestin isolated from bovine rod outer segments. Protein Sci 3:314-324.

Peterson JJ, Tam BM, Moritz OL, Shelamer CL, Dugger DR, McDowell JH, Hargrave PA, Papermaster DS, Smith WC (2003) Arrestin migrates in photoreceptors in response to light: a study of arrestin localization using an arrestin-GFP fusion protein in transgenic frogs. Exp Eye Res 76:553-563.

Rajan RS, Illing ME, Bence NF, Kopito RR (2001) Specificity in intracellular protein aggregation and inclusion body formation. Proc Natl Acad Sci USA 98:13060-13065.

Robinson PR, Cohen GB, Zhukovsky EA, Oprian DD (1992) Constitutively active mutants of rhodopsin. Neuron 9:719-725.

Roca A, Shin KJ, Liu X, Simon MI, Chen J (2004) Comparative analysis of transcriptional profiles between two apoptotic pathways of light-induced retinal degeneration. Neuroscience 129:779-790.

Satoh AK, Ready DF (2005) Arrestin1 mediates light-dependent rhodopsin endocytosis and cell survival. Curr Biol 15:1722-1733.

Schremser JL, Williams TP (1995) Rod outer segment (ROS) renewal as a mechanism for adaptation to a new intensity environment. I. Rhodopsin levels and ROS length. Exp Eye Res 61:17-23.

Shi GW, Chen J, Concepcion F, Motamedchaboki K, Marjoram P, Langen R (2005) Light causes phosphorylation of non-activated visual pigments in intact mouse rod photoreceptor cells. J Biol Chem

Shi W, Sports CD, Raman D, Shirakawa S, Osawa S, Weiss ER (1998) Rhodopsin arginine-135 mutants are phosphorylated by rhodopsin kinase and bind arrestin in the absence of 11-cis-retinal. Biochemistry 37:4869-4874.

Smith WC (1996) A splice variant of arrestin from human retina. Exp Eye Res 62:585-592.

Smith WC, Milam AH, Dugger D, Arendt A, Hargrave PA, Palczewski K (1994) A splice variant of arrestin. Molecular cloning and localization in bovine retina. J Biol Chem 269:15407-15410.

Sung CH, Davenport CM, Nathans J (1993) Rhodopsin mutations responsible for autosomal dominant retinitis pigmentosa. Clustering of functional classes along the polypeptide chain. J Biol Chem 268:26645-26649.

Sung CH, Makino C, Baylor D, Nathans J (1994) A rhodopsin gene mutation responsible for autosomal dominant retinitis pigmentosa results in a protein that is defective in localization to the photoreceptor outer segment. J Neurosci 14:5818-5833.

Tam BM, Moritz OL, Hurd LB, Papermaster DS (2000) Identification of an outer segment targeting signal in the $\mathrm{COOH}$ terminus of rhodopsin using transgenic Xenopus laevis. J Cell Biol 151:1369-1380.

Tsang SH, Burns ME, Calvert PD, Gouras P, Baylor DA, Goff SP, Arshavsky VY (1998) Role for the target enzyme in deactivation of photoreceptor G protein in vivo. Science 282:117-121.

Vishnivetskiy SA, Schubert C, Climaco GC, Gurevich YV, Velez MG, Gurevich VV (2000) An additional phosphate-binding element in arrestin molecule. Implications for the mechanism of arrestin activation. J Biol Chem 275:41049-41057.

Whelan JP, McGinnis JF (1988) Light-dependent subcellular movement of photoreceptor proteins. J Neurosci Res 20:263-270.

Wilden U (1995) Duration and amplitude of the light-induced cGMP hydrolysis in vertebrate photoreceptors are regulated by multiple phosphorylation of rhodopsin and by arrestin binding. Biochemistry 34:1446-1454 .

Wilden U, Kuhn H (1982) Light-dependent phosphorylation of rhodopsin: number of phosphorylation sites. Biochemistry 21:3014-3022.

Wilden U, Hall SW, Kuhn H (1986) Phosphodiesterase activation by photoexcited rhodopsin is quenched when rhodopsin is phosphorylated and binds the intrinsic 48 -kDa protein of rod outer segments. Proc Natl Acad Sci USA 83:1174-1178.

Xu J, Dodd RL, Makino CL, Simon MI, Baylor DA, Chen J (1997) Prolonged photoresponses in transgenic mouse rods lacking arrestin. Nature 389: 505-509.

Zhukovsky EA, Robinson PR, Oprian DD (1991) Transducin activation by rhodopsin without a covalent bond to the 11-cis-retinal chromophore. Science 251:558-560. 